\title{
NOTA SOBRE ONOCLEOPSIS HINTONII (ONOCLEACEAE), UN NUEVO REGISTRO PARA EL ESTADO DE HIDALGO, MÉXICO
}

Felipe Gómez-Noguez ${ }^{1,2}$, Aniceto Mendoza-Ruiz ${ }^{1}$ y Blanca Pérez-García ${ }^{1}$

${ }^{1}$ Universidad Autónoma Metropolitana-Iztapalapa, Departamento de Biología, Laboratorio de Biología de Pteridofitas, Área de Botánica Estructural y Sistemática Vegetal, Avenida San Rafael Atlixco 186, Colonia Vicentina, Delegación Iztapalapa, 09340 México, D.F., México.

${ }_{2}^{2}$ Autor para la correspondencia: tindalo129@gmail.com

\section{RESUMEN}

Se reporta por primera vez a Onocleopsis hintonii para el estado de Hidalgo, México, adicionando el registro de la familia Onocleaceae Pic. Serm. Se presentan datos relativos a su morfología y las características de sus esporas.

Palabras clave: Hidalgo, México, nuevo registro, Onocleaceae, Onocleopsis.

\section{ABSTRACT}

Onocleopsis hintonii is reported for the first time for the state of Hidalgo, Mexico, adding the Onocleaceae Pic. Serm. family record. Data on their morphology and characteristics of their spores are presented.

Key words: Hidalgo, Mexico, new record, Onocleaceae, Onocleopsis.

Dentro del proyecto de tesis de maestría del primer autor, en el Municipio de Molango de Escamilla y sus alrededores, se hizo trabajo de campo de febrero del 2009 a marzo del 2010, realizando 12 excursiones a la Sierra Hidalguense, en la localidad de Río Malila, en donde se recolectó material biológico, en particular pteridofitas. En una exploración dentro del bosque, se encontró creciendo entre las rocas y a lo largo de un arroyo, una población de helechos con hojas dimórficas, que 
resultó corresponder a Onocleopsis hintonii F. Ballard. Después de hacer una revisión bibliográfica y consultar los herbarios ENCB, FCME, MEXU y UAMIZ, no se hallaron registros de esta especie para el estado de Hidalgo.

La población es pequeña, se encuentra en el fondo de un barranco en un fragmento de bosque mesófilo de montaña (BMM), en donde existen afloramientos de rocas sedimentarias (calizas y lutitas) del Jurásico Superior; predominan los suelos luvisoles órticos y cambisoles éutricos; el clima es de tipo C(fm)-b(e)g (RamírezTrejo et al., 2004). La flora vascular del lugar, en el estrato arbóreo, se encuentra caracterizada por Liquidambar styraciflua L. y Quercus L., en el sotobosque se desarrollan numerosas herbáceas y entre ellas sobresalen otras pteridofitas como Adiantum andicola Liebm., Asplenium abscissum Willd., Cystopteris fragilis (L.) Berhn., Dennstaedtia globulifera (Poir.) Hieron., Diplazium lonchophyllum Kunze, Holodictyum ghiesbreghtii (E. Fourn.) Maxon, Llavea cordifolia Lag., Pellaea ovata (Desv.) Weath., Selaginella pallescens (C. Presl) Spring, Tectaria heracleifolia (Willd.) Underw. y Woodwardia martinezii Maxon ex Weath.; otros helechos como Campyloneurum angustifolium (Sw.) Fée, C. xalapense Fée, Pecluma sursumcurrens (Copel.) M. G. Price, Pleopeltis crassinervata (Fée) T. Moore, Polypodium lepidotrichum (Fée) Maxon, y P. plebeium Schltdl. \& Cham., se encuentran como epífitas sobre los troncos de árboles de la zona.

La ubicación taxonómica del género monotípico Onocleopsis F. Ballard ha variado de acuerdo con el criterio de los autores; ha sido considerado dentro de las Woodsiaceae (Riba, 1995; Tejero-Díez y Arreguín-Sánchez, 2004), Dryopteridaceae (Gastony y Ungerer, 1997) o Aspleniaceae (Tahuilán et al., 2003). Sin embargo, Smith et al. (2006) lo incluyen como uno de los cuatro géneros que conforman a las Onocleaceae Pic. Serm., ante lo cual se reconoce una nueva familia para el estado de Hidalgo.

Onocleopsis hintonii es una especie poco común, propia de bosques mesófilos de montaña (BMM). Históricamente, fue encontrada por primera vez en Oaxaca por Conzatti \& González en 1847 (Ballard, 1948), sin embargo la descripción fue hecha por Ballard, a partir de un ejemplar herborizado recolectado por Hinton en el municipio de Temascaltepec, Estado de México (Ballard, 1945). Posteriormente ha sido registrada para los estados de Guerrero, Oaxaca, Morelos (Tryon y Tryon, 1982; Riba et al., 1996), Jalisco, Michoacán, Puebla (Tahuilán et al., 2003, Mickel y Smith, 2004) y Chiapas (Tejero-Díez y Arreguín-Sánchez, 2004). El hallazgo de la misma en la sierra hidalguense, aislada de las localidades conocidas en la vertiente del Pacífico y centro de México, plantea la posibilidad del desarrollo de una variedad o forma para la Sierra Madre Oriental, razón por la cual se revisó el material 
depositado en los herbarios, se confrontó con el recolectado en Hidalgo y se elaboró la descripción de la especie a fin que sea más completa e incluya las variaciones de las poblaciones.

El material recolectado (esporas), junto con los ejemplares de respaldo ( $F$. Gómez N.-273 y F. Gómez N.-282), se encuentran depositados en el Herbario Metropolitano "Dr. Ramón Riba y Nava Esparza” (UAMIZ) de la Universidad Autónoma Metropolitana, Unidad Iztapalapa. La planta se encontró aproximadamente a 200 metros adentro del bosque al lado de la carretera federal 105 Pachuca - Tampico, kilómetro 118, entre el rancho San Antonio y la Finca Casaigne, cercanas al Río Malila, con coordenadas $20^{\circ} 43^{\prime} 21^{\prime \prime} \mathrm{N}$ y 98 $43^{\prime} 36^{\prime \prime} \mathrm{W}$, a una altitud de $1507 \mathrm{~m}$, en BMM.

Onocleopsis hintonii es una planta terrestre, con rizoma erecto de hasta $1 \mathrm{~m}$ de alto, firme, a veces estolonífero en la base y con escamas en el ápice; éstas son linear lanceoladas y largamente atenuadas, de 1 a $4 \mathrm{~mm}$ de ancho por 6 a $28 \mathrm{~mm}$ de largo, son de color pardo claro a obscuro (Fig. 1B). Hojas dimórficas de 50 a 130 cm de largo (Fig. 1A); el pecíolo mide de 2 a $5 \mathrm{~mm}$ de diámetro, es de color pajizo y escamoso adaxialmente, las escamas miden de 1 a $3 \mathrm{~mm}$ de ancho por 13 a $18 \mathrm{~mm}$ de largo; la lámina de la hoja estéril es pinnada, ovado lanceolada, mide de 33 a $130 \mathrm{~cm}$ de largo y de 30 a $45 \mathrm{~cm}$ de ancho, su ápice es pinnatífido, el raquis es escasamente escamoso, las escamas se limitan a la base de la cara adaxial; presenta de 13 a 25 pares de pinnas, alternas a opuestas, enteras a lobadas, las pinnas miden de 15 a 24 cm de largo por 2.6 a $4.5 \mathrm{~cm}$ de ancho, con margen dentado a aserrado; las pinnas basales se encuentran reducidas en tamaño y con una aurícula acroscópica (Fig. 1C), el ápice de las pinnas es largamente acuminado a cuneado, y la venación es reticulada, la vena media está flanqueada por areolas grandes que se extienden entre las venas secundarias (Fig. 1D); la hoja fértil presenta escaso tejido laminar (Figs. 1E y $1 \mathrm{~F})$, de 10 a $21 \mathrm{~cm}$ de ancho y de 68 a $130 \mathrm{~cm}$ de largo, la lámina es 3 pinnada, las pinnas sulcadas en la cara adaxial, con numerosas escamas adaxialmente sólo en la base de las pinnas. Los soros se encuentran envueltos por un tejido papiráceo de color blanco, que corresponde al indusio (Fig. 1F), y constituyen el último segmento de la hoja fértil. Las esporas son monoletes y clorofílicas, de 48 x $65 \mu \mathrm{m}$ en promedio, presentan perisporio de $8 \mu \mathrm{m}$ de grosor, de ornamentación reticulada, ligeramente plegado y flácidamente rodeando a la exina (Fig. 1G), número cromosómico $n=40$.

Las características generales de las esporas de Onocleopsis hintonii fueron descritas por Lloyd (1971). En el análisis L-O concordamos con lo descrito por Kato y Sahashi (1980), el perisporio presenta una superficie irregularmente equinada sobre estructuras similares a muri de $1 \mu \mathrm{m}$ de alto y de $0.5 \mu \mathrm{m}$ de ancho, separadas por áreas similares a lúmenes de diámetro inferior a $2 \mu \mathrm{m}$. Utilizando microscopía 

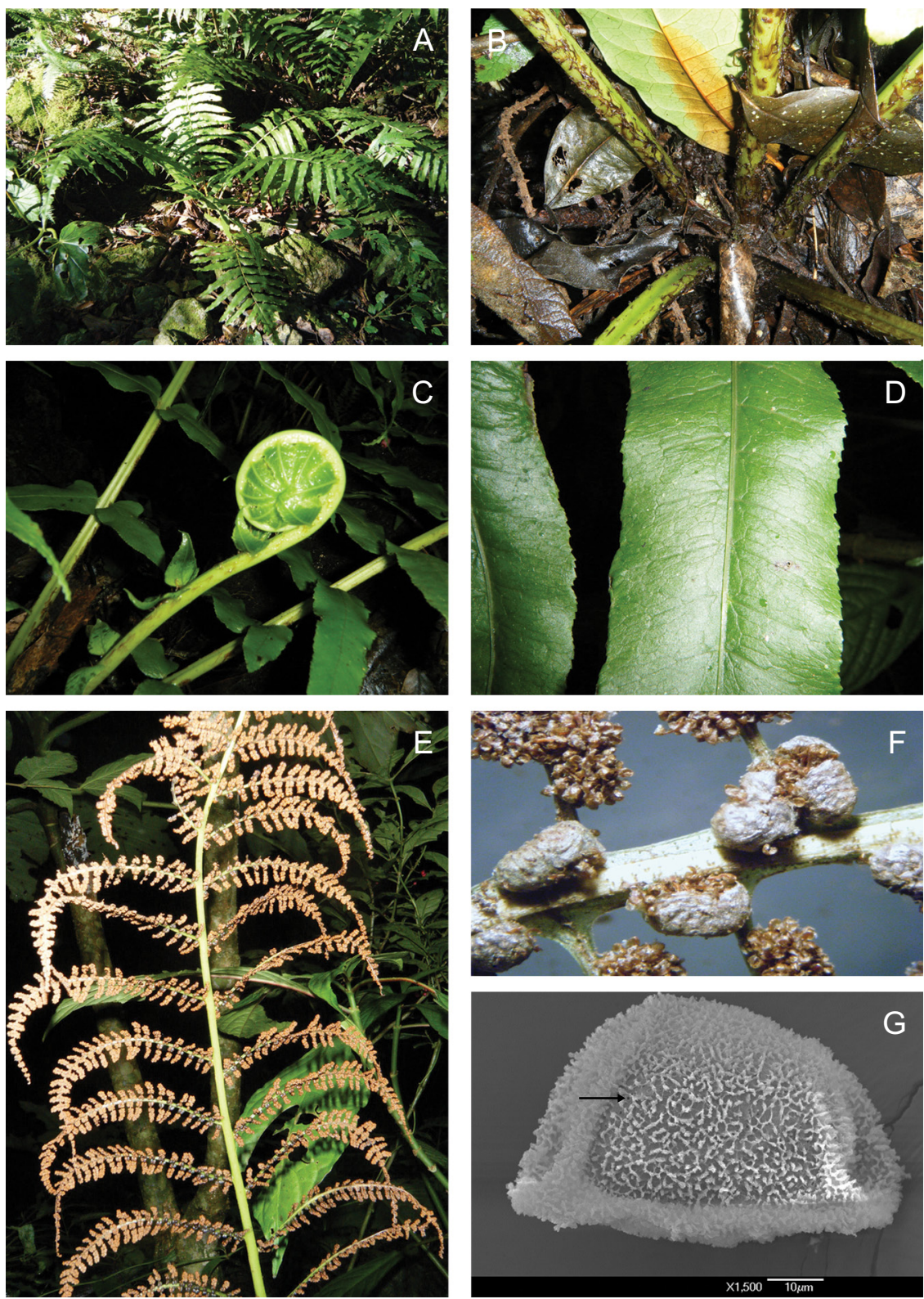

Fig. 1. Onocleopsis hintonii. A. planta en su hábitat; B. detalle de la base del pecíolo; C. primordio foliar circinado; D. fragmento de pinna estéril con venación reticulada y margen aserrado; E. hoja fértil madura; F. detalle de las pinnas fértiles, nótese el indusio vestigial 6x. G. espora monolete, vista ecuatorial (MEB) se muestra una ornamentación de retículo incompleto con cristas en los muri (reticulum cristatum), y algunos foveolos (flecha). 
electrónica de barrido (MEB), las características de la espora concuerdan con las descritas por Tryon y Lugardon (1990), quienes mencionan la presencia de un perisporio cavado con la parte interna papilada y la superficie con equinas distribuidas uniformemente sobre los pliegues toscos e irregulares; sin embargo en la figura $1 \mathrm{G}$ se puede apreciar que dichos pliegues son rectos con un patrón geométrico, presente en las vistas distal y ecuatorial; además se aprecian foveolos presentes en el perisporio. Estas diferencias puede deberse a la fuente del material examinado, obtenido de muestras de herbario en los trabajos previos, y tomado de material fresco para el presente estudio.

A pesar de que se han llevado acabo estudios pteridológicos recientes que han aportado nuevos registros para la entidad (Luna et al., 1994; Alcántara y Luna, 2001; Ponce-Vargas et al., 2006; Cuevas-Hernández y Sánchez-González, 2009; Mendoza-Ruiz y Pérez-García, 2009; Ramírez-Cruz et al., 2009; Ceja-Romero et al., 2010), que adicionados a los 12 consignados para el municipio de Tenango de Doria (Sánchez-González, com. pers.) y el aquí señalado alcanzan la cifra de 314 especies, agrupadas en 73 géneros y 29 familias, aún hace falta trabajo de campo para tener un mejor conocimiento de la pteridoflora del estado.

La carencia de registros de $O$. hintonii en la zona de estudio puede estar relacionada con la falta de colectas en la región, pero también con el estado incipiente de la población, ya que por el tamaño del rizoma del material recolectado cabe la posibilidad de que se trate de una población joven.

De acuerdo con Riba (1995) el intervalo altitudinal registrado para la especie es de los 1300 a los $2800 \mathrm{~m}$, creciendo siempre en barrancas o cañadas húmedas, cerca de afluentes de agua, a lo largo de riachuelos y/o debajo de caídas de agua. Podría ser posible encontrar otras poblaciones de $O$. hintonii en otras zonas de la sierra hidalguense con orografía parecida, dentro de la subprovincia Carso-Huasteco, donde se hallan numerosas laderas abruptas, cañones y lomeríos, como en los municipios de Tlanchinol, Xochicoatlán, Calnali, entre otros; región que de acuerdo con Sanginés-Franco et al. (2011) mostró una mayor riqueza de helechos en la provincia de la Sierra Madre Oriental.

La distribución de esta especie en BMM, aunada a la rareza del hallazgo, el bajo número de individuos y poblaciones; así como a su singular exclusividad para México y Guatemala es sustento para su futura inclusión en la Norma Oficial Mexicana NOM-059-SEMARNAT-2010 (Protección ambiental-Especies nativas de México de flora y fauna silvestres-Categorías de riesgo y especificaciones para su inclusión, exclusión o cambio-Lista de especies en riesgo) bajo la categoría "amenazada" (Anónimo, 2010). 


\section{AGRADECIMIENTOS}

Al Consejo Nacional de Ciencia y Tecnología (beca 224680) al programa de Posgrado de la Universidad Autónoma Metropolitana-Unidad Iztapalapa, a los Bióls. Karla Castillo y Ricardo Valdez así como al Ing. A. Iván Gómez por el apoyo en el trabajo de campo; a la Dra. Silvia Espinosa Matías por el procesamiento de muestras y obtención de imágenes de MEB de esporas, a la M. en C. Ana Rosa López-Ferrari por sus comentarios y sugerencias para enriquecer el manuscrito; a la Biól. Rosa María Murillo por la revisión crítica del manuscrito y al personal de los herbarios ENCB, FCME, MEXU y UAMIZ. A los revisores anónimos por sus valiosos comentarios y sugerencias.

\section{LITERATURA CITADA}

Alcántara, A. y I. Luna V. 2001. Análisis florístico de dos áreas con bosque mesófilo de montaña en el estado de Hidalgo, México: Eloxochitlán y Tlahuelompa. Acta Bot. Mex. 54: 51-87.

Anónimo. 2010. Norma Oficial Mexicana NOM-059-SEMARNAT-2010 (Protección ambiental-Especies nativas de México de flora y fauna silvestres-Categorías de riesgo y especificaciones para su inclusión, exclusión o cambio-Lista de especies en riesgo). Consultado el 26 de julio de 2011. http://www.profepa.gob.mx/innovaportal/ file/435/1/NOM_059_SEMARNAT_2010.pdf

Ballard, F. 1945. A new fern genus from Mexico and Guatemala. Amer. Fern J. 35(1): 1-3.

Ballard, F. 1948. Further Notes on Onocleopsis. Amer. Fern J. 38(4): 125-132.

Ceja-Romero, J., A. Mendoza-Ruiz, A. R. López-Ferrari, A. Espejo-Serna, B. Pérez-García y J. García-Cruz. 2010. Las epífitas vasculares del estado de Hidalgo, México: Diversidad y distribución. Acta Bot. Mex. 93: 1-39.

Cuevas-Hernández, A. L. y A. Sánchez-González. 2009. Contribución al estudio florístico de helechos y selaginelas de la Reserva de la Biósfera Barranca de Metztitlán, estado de Hidalgo, México. Memorias del VII Congreso Nacional sobre áreas Naturales Protegidas de México, San Luis Potosí. Consultado el 26 de mayo 2011. http://www. congresoanps.gob.mx/

Gastony, G. J. y M. C. Ungerer. 1997. Molecular systematic and revised taxonomy of the onocleid ferns (Dryopteridaceae: Onocleeae). Amer. J. Bot. 84(6): 840-849.

Kato, M. y N. Sahashi. 1980. Affinities in the onocleoid ferns. Acta Phytotax. Geobot. 31(46): 127-138.

Lloyd, R. M. 1971. Systematics of the onocleoid ferns. Univ. Calif. Publ. Bot. 61: 1-93.

Luna, V. I., S. Ocegueda C. y O. Alcántara A. 1994. Florística y notas biogeográficas del bosque mesófilo del municipio de Tlanchinol, Hidalgo, México. Anales Inst. Biol. Univ. Nac. Aut. México, Ser. Bot. 65(1): 31-62. 
Mendoza-Ruiz, A. y B. Pérez-García. 2009. Helechos y licopodios de México Vol. I. Comisión Nacional para el Conocimiento y Uso de la Biodiversidad, Universidad Autónoma Metropolitana. México. D.F., México. 287 pp.

Mickel, J. T. y A. R. Smith. 2004. The pteridophytes of Mexico. Mem. New York Bot. Gard. 88: 1-1054.

Ponce-Vargas, A., I. Luna-Vega, O. Alcántara-Ayala y C. A. Ruiz-Jiménez. 2006. Florística del bosque mesófilo de montaña de Monte Grande, Lolotla, Hidalgo, México. Rev. Mex. Biodiv. 77: 177-190.

Ramírez-Cruz, S., A. Sánchez-González y D. Tejero-Díez. 2009. La pteridoflora del Parque Nacional Los Mármoles, Hidalgo, México. Bol. Soc. Bot. Méx. 84: 35-44.

Ramírez-Trejo, R., B. Pérez-García y A. Orozco-Segovia. 2004. Analysis of fern spore banks from the soil of three vegetation types in the central region of Mexico. Amer. J. Bot. 91(5): 682-688.

Riba, R. 1995. Onocleopsis F. Ballard. In: Moran, R. C. \& R. Riba (eds.). Flora Mesoamericana. Vol. I. Psilotaceae a Salviniaceae. Instituto de Biología, Universidad Nacional Autónoma de México, Missouri Botanical Garden y The Natural History Museum. México, D. F., México. pp. 246-247.

Riba, R., L. Pacheco, A. Valdés y Y. Sandoval. 1996. Pteridoflora del estado de Morelos, México. Lista de familias, géneros y especies. Acta Bot. Mex. 37: 45-65.

Sanginés-Franco, C., I. Luna-Vega, O. Alcántara A. y R. Contreras-Medina. 2011. Distributional patterns and biogeographic analysis of ferns in the Sierra Madre Oriental. Amer. Fern J. 101(2): 81-104.

Smith, A. R., K. M. Pryer, E. Schuettpelz, P. Korall, H. Schneider y P. G. Wolf. 2006. A classification for extant ferns. Taxon 55(3): 705-731.

Tahuilán M, J. U., M. L. Arreguín-Sánchez y R. Fernández-Nava. 2003. Revisión de los géneros Onocleopsis, Plecosorus y Polystichum (Aspleniaceae-Pteridophyta) para la cuenca del río Balsas, México. Polibotánica 15: 113-146.

Tejero-Díez, D. y M. L. Arreguín-Sánchez. 2004. Lista con anotaciones de los pteridófitos del estado de México. Acta Bot. Mex. 69: 1-82.

Tryon, A. F. y B. Lugardon. 1990. Spores of the Pteridophyta: surface, wall structure and diversity based on electron microscope studies. Springer-Verlag. Nueva York, USA. $647 \mathrm{pp}$.

Tryon, R. M. y A. F. Tryon. 1982. Fern and allied plants with special reference to tropical America. Springer-Verlag. New York, USA. 857 pp. 